\title{
Research progress of polydiacetylene sensing materials in food biotechnology
}

\author{
Hui Wang ${ }^{1 *}$, Li Han ${ }^{1}$, Weijuan Gong ${ }^{1}$ and Ranran Chu ${ }^{1}$ \\ ${ }^{1}$ Department of chemistry, Qilu Institute of Technology, Jinan City, Shandong Province, 250000, China
}

\begin{abstract}
Polydiacetylene (PDAs) is a special semiconductor polymer with an alternating conjugated structure of ene-acetylene and unique optical and electronic properties. Typically, PDAs change from blue to red in response to the external stimuli, such as temperature, $\mathrm{pH}$, ions, organic solvents, pressure, and donor-acceptor interactions. This colorimetric response of PDAs makes them one of the excellent materials for chemical or biosensor analysis platforms. In this review, we briefly describe the research progress of polydiacetylene systems in the field of food biotechnology. The challenges and outlooks of PDAs sensing materials in visual detection of preservatives in food innovatively were also discussed.
\end{abstract}

\section{Introduction}

Polydiacetylene (PDAs) is a special semiconductor polymer with an alternating conjugated structure of ene-acetylene, and $\pi$-electron delocalization in the whole conjugated system main chain. Therefore, PDAs has unique optical and electronic properties (Figure 1). The appearance color of polydiacetylene is related to the conformation of its main chain. The most common color is "blue" $(\lambda \max \approx 620 \mathrm{~nm})$, which corresponds to the PDAs structure with the longest effective conjugate length. The second is "red" $(\lambda \max \approx 550 \mathrm{~nm})$, of which the backbone conformation of the polymer is a little distorted. "Purple" and "yellow" have also been reported ${ }^{[1,2]}$. Among the various color phases of PDAs, blue PDAs are relatively unstable, and obvious color changes will occur when exposed to external stimuli. Environmental stimuli can take various forms, such as temperature (thermochromic), $\mathrm{pH}$ (acid-base), ions, organic solvents (solvochromic), pressure (mechanical chromic), and donor-acceptor interactions ${ }^{[3-9]}$. Typically, PDAs change from blue to red in response to these external stimuli. The corresponding UV-vis absorption spectrum will show a decrease in absorption at the long wavelength $(620 \mathrm{~nm})$ and an increase in absorption at the short wavelength $(550 \mathrm{~nm})$. These colorimetric transitions can be observed with the naked eye and have been used in the development of PDAs-based colorimetric sensor systems.

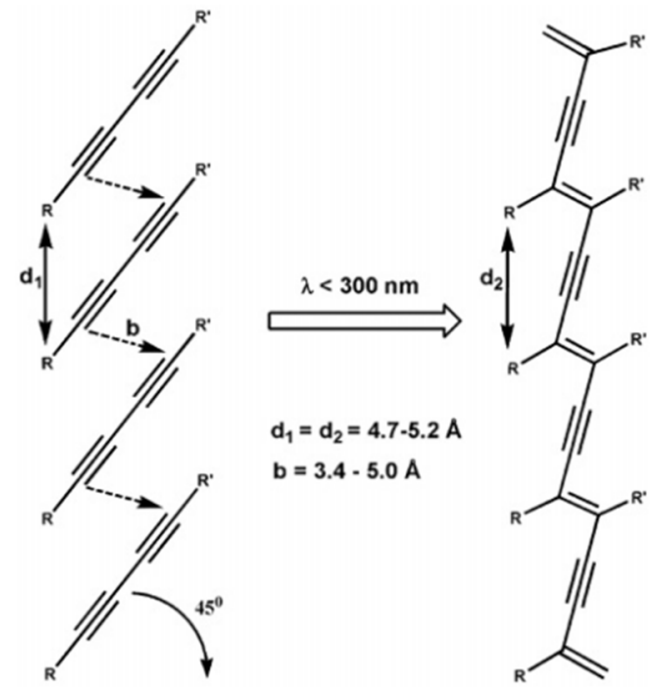

Figure 1 Polymerization of diacetylene under ultraviolet radiation

This colorimetric response of PDAs makes them one of the excellent materials for chemical or biosensor analysis platforms. In recent years, many biological and chemical sensors based on the optical response of polydiacetylene have been reported ${ }^{[10-12]}$. Thermochromic and solvent-chromic properties of PDAs are mostly studied. However, thermochromic temperature is relatively high, and most of the solvents used in the study of solvent-chromic properties are organic solvents with pungent odor, volatile and toxic. The roots of the colorimetric shift are not fully understood. Understanding the basis of PDAs variation is necessary for the design of sensitive, selective and stable PDAs. Polymerization of diacetylene molecules transfers the hybridization of carbon atoms on alkynes from $\mathrm{sp}$ hybridization to $\mathrm{sp}^{2}$ hybridization, thus changing the 
bond angle from $180^{\circ}$ to $120^{\circ}$. As the polymerization rate increases, the accumulation of side chains prevents the rearrangement of the main chains of the polymer, which results in the accumulation of stress in the self-assembled structure. However, under the action of external stimulus, the stress on the side chain in the system may be released, which causes the main chain of the polymer to rearrange, conformational change, and then show the corresponding color change $^{[13]}$. In addition, red PDAs also have weak red fluorescence. The optical color change signal of the system is not as strong as that of the conventional dye labeled sensor system, but PDAs is very convenient and effective system, they can be applied to the unlabeled sensor system.

\section{Research progress of polydiacetylene sensing materials}

Introducing receptor units with special functions into PDAs system can realize the sensing of specific analytes. In this method, the receptor part is covalently attached to the monomer biacetylene unit, and then directly self-assembled or co-assembled after mixing with biacetylene monomer, and then polymerized to obtain the PDAs sensor. Although this method requires the chemical synthesis process to introduce the receptor part into the PDAs matrix, but the stability and sensing performance of the final PDAs system are good. Thus the method is still widely used.

$\mathrm{He}^{[14]}$ developed a biosensor based on polydiacetylene for detection of $\mathrm{H} 5$ influenza virus (Figure 2). A stable monomer DA vesicle was prepared by mixing 10,12-pentacosadiynoicacid (PCDA) with 1 , 2-dimyrisoyl-sn-glyceryl-3-choline phosphate (DMPC) at the ratio of $6: 4$. After polymerization, the surface of the vesicle was activated with NHS/EDC and then attached to the anti-HA-mAb, which was subsequently sealed with BSA. When the H5 influenza virus was added, the biosensor showed a visible blue to red color shift. The response did not occur in the presence of $\mathrm{H} 3$ influenza virus, Newcastle disease virus and porcine reproductive and respiratory syndrome virus. These observations indicate that the sensor has good selectivity to H5 influenza virus. In addition, the detection limit of this sensor for H5 influenza virus is about 0.53 copies $/ \mu \mathrm{L}$. Compared with traditional methods (virus isolation and real-time polymerase chain reaction), this new PDAs-based sensing system is very accurate in the quantification of viruses.

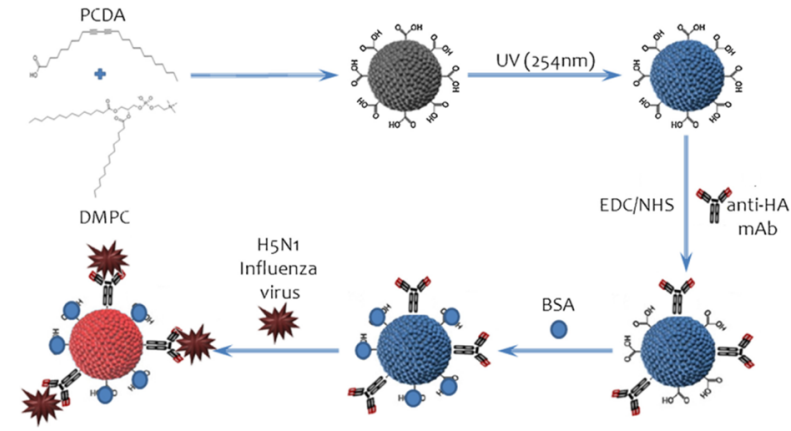

Figure 2 Schematic diagram of the preparation of the H5 influenza virus biosensor based on PDAs

Jeong $^{[15]}$ designed a PDAs system conjugated with M149 antibody, and modified PCDA vesicles with M149 antibody. Antibody-antigen biomolecular recognition could realize the transformation of PDAs conformation, thus converting biological signals into optical signals, so as to visually detect influenza viruses (Figure 3). PCDA vesicle is coated on polyvinylidene fluoride (PVDF) membrane to form a membrane-type sensing platform can improve the stability and portability of the vesicle system.

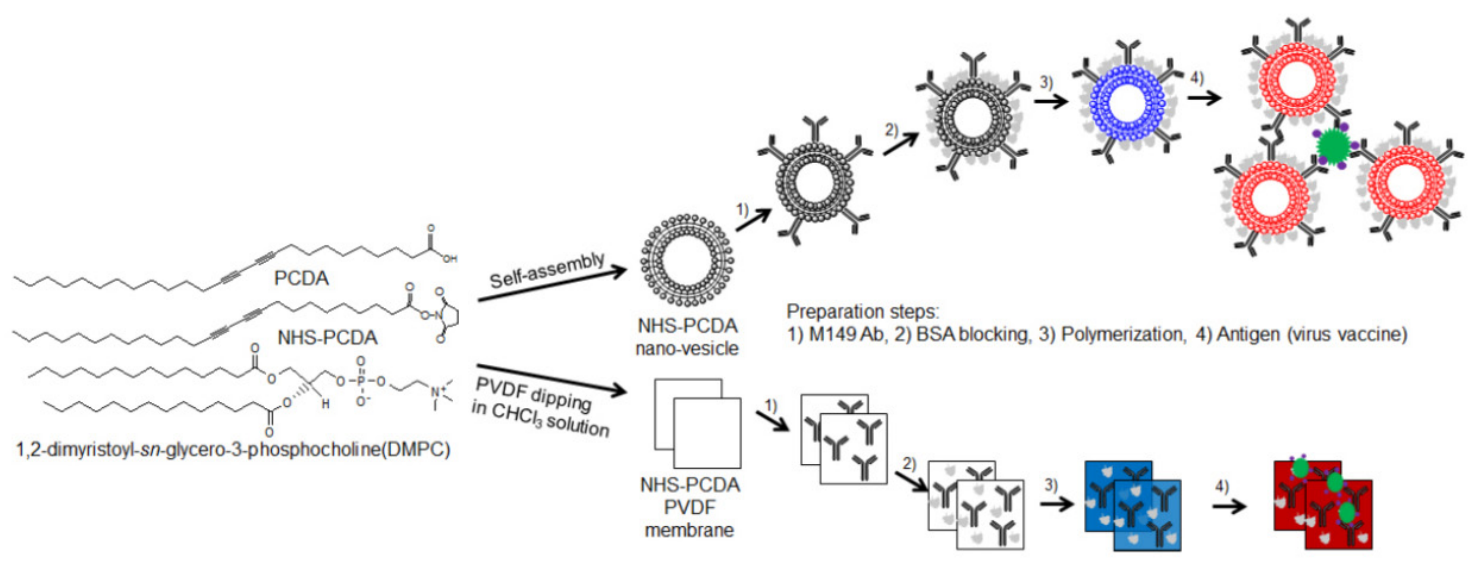

Figure 3 PDAs system conjugated with M149 antibody for detection of influenza virus

Zhou $^{[16]}$ combined target-specific aptamers with PDAs, then, the aptam-conjugated PDAs vesicles were coated on PVDF strips by simple solvent evaporation. A colorimetric PDAs test paper sensor was prepared to visually identify Bacillus Thuringiensis (BT) HD-73 spores by the unique blue-red transition of PDAs (Figure 4). The response time of the strip is less than 1 hour, and the detection limit is $3 \times 10^{7} \mathrm{CFU} / \mathrm{mL}$, which is far lower than the level harmful to human body. 


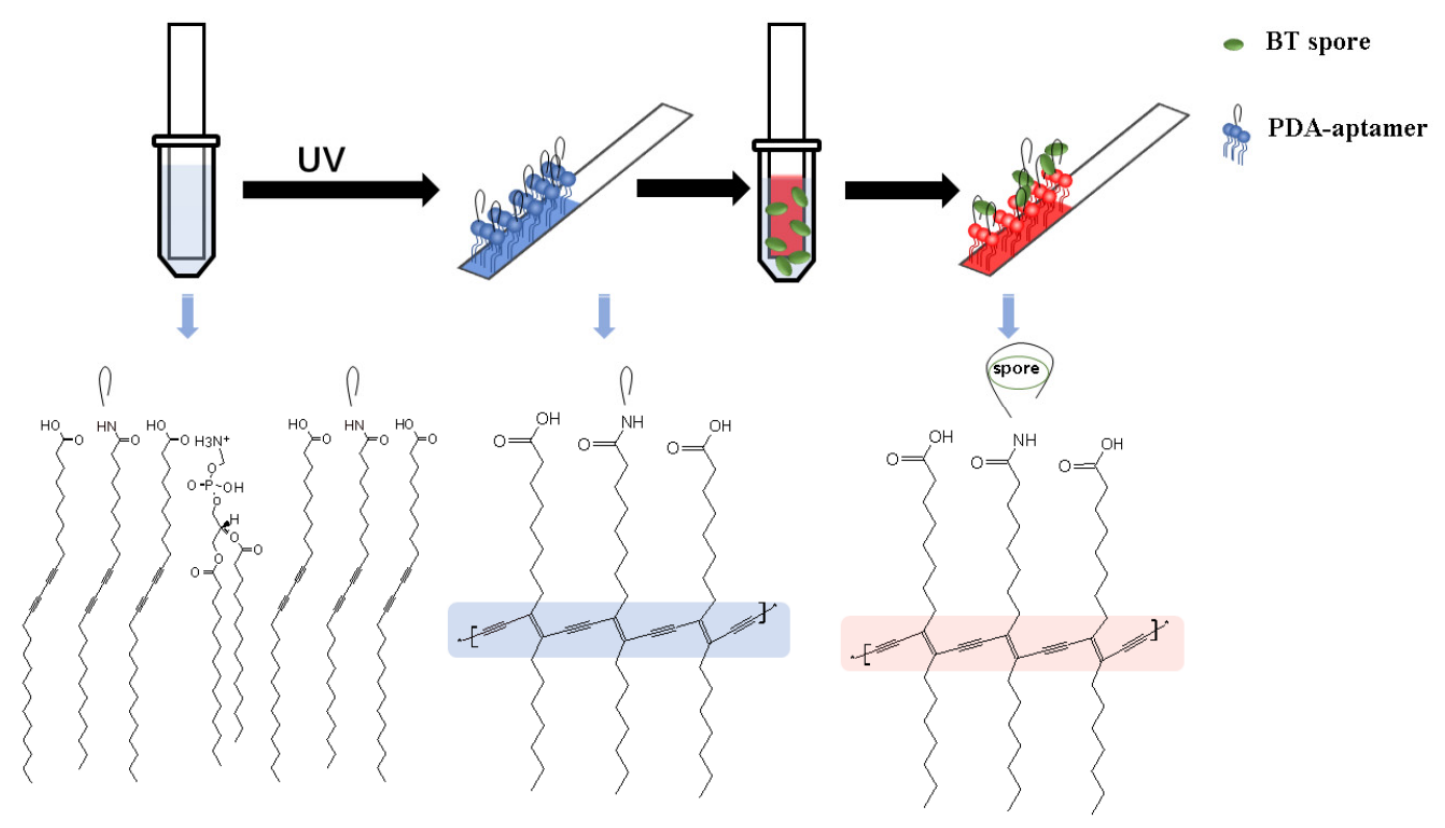

Figure 4 Schematic diagram of visual recognition of Bacillus Thuringiensis by specific aptamer modified PDAs system

Benzoic acid, is not soluble in water, and has obvious inhibitory effect on bacteria, mold, yeast and other microorganisms under acidic conditions. It is one of the commonly used preservatives in food. Due to its insolubility, most of its sodium salts are used in the actual production process, and the sodium benzoate in food is converted into benzoic acid after dissolution to produce bacteriostatic effect ${ }^{[17]}$. At present, sodium benzoate is allowed to be used as food preservative in many countries. Because of its low price, it is still widely used as a preservative in the processing and production of various kinds of food in China. Sodium benzoate in the safe dose range will not cause harm to human health, but excess sodium benzoate is converted into benzoic acid under the action of gastric acid, thus causing great harm to the liver. Therefore, the determination of sodium benzoate content in food is particularly important. At present, the content determination methods of sodium benzoate are mainly high performance liquid chromatography, gas chromatography and thin layer chromatography, etc. ${ }^{[18-21]}$. These methods are simple, rapid and accurate, but the operation is relatively complex and the required equipment is expensive. Therefore, it is necessary to develop a simple, economical, high sensitivity and high selectivity method for the determination of sodium benzoate.

$\mathrm{Xue}^{[22]}$ combined a dispersion-liquid microextraction technique with surface-enhanced Raman scattering to detect sodium benzoate, a preservative in oral ibuprofen solutions. This detection can be finished within 10 minutes, and the detection limit is $0.56 \mathrm{mg} / \mathrm{L}$. A vesicle probe was prepared by connecting diacetylene monomer with benzoic acid hapten, p-aminobenzoic acid, according to the optical properties of polydiacetylene vesicles that change from blue to red when disturbed by the outside world by Meng ${ }^{[23]}$. The blue vesicles appear red when specifically combined with antibodies to benzoic acid, and the vesicles change from red to blue after contact with benzoic acid. A non-labeled homogeneous colorimetric method for the detection of sodium benzoate was established (Figure 5).

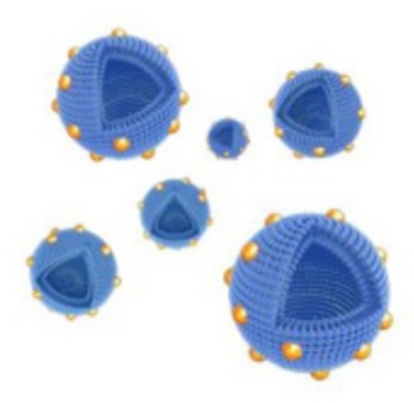

Blue (dispersed) Phase
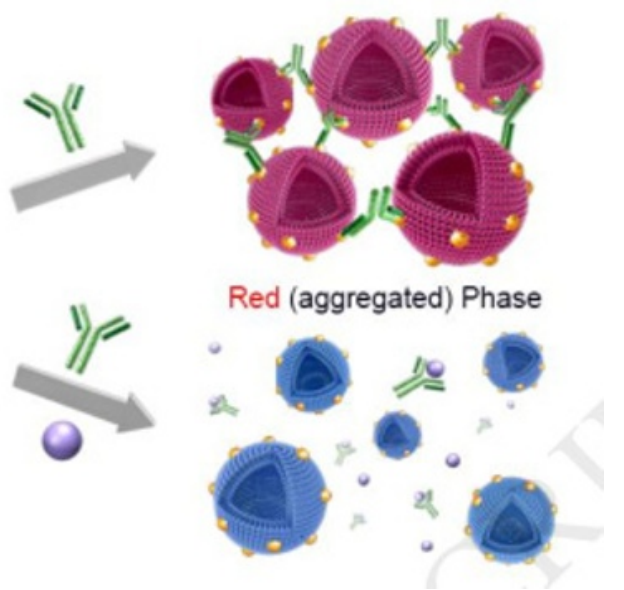

Figure 5 Schematic diagram of detection of benzoic acid by PDAs vesicles modified with benzoic acid haptens 


\section{Conclusion}

At present, the research of introducing specific antibodies into PDAs system to realize visual detection of viruses and bacteria has been quite mature. However, there are few studies on the colorimetric detection of food preservatives using PDAs system, and most of them are performed using PDAs vesicles for detection. The relative stability of vesicles is poor, and the vesicles are not easy to carry. Based on the above research status, it is necessary to further expand the application scope of PDAs system, and make use of the colorimetric property of PDAs system and the portability and stability of the film to realize the rapid and visual detection of preservatives in food innovatively.

\section{Reference}

1. Filhol, J. S.; Deschamps, J.; Dutremez, S. G.; Boury, B.; Barisien, T.; Legrand, L.; Schott, M. Polymorphs and colors of polydiacetylenes: a first principles study. J. Am. Chem. Soc. 2009, 131, 6976-6988.

2. Diegelmann, S. R.; Tovar, J. D. Polydiacetylene-peptide 1D nanomaterials. Macromol.Rapid Commun. 2013, 34, 1343-1350.

3. Qian, X.; Städler, B. Recent developments in polydiacetylene-based sensors. Chem. Mater. 2019, 31, 1196-1222.

4. Weston, M.; Tjandra, A. D.; Chandrawati, R. Tuning chromatic response, sensitivity, and specificity of polydiacetylene-based sensors. Polym. Chem. 2020, 11, 166-183.

5. Fang, F.; Meng, F.; Luo, L. Recent advances on polydiacetylene-based smart materials for biomedical applications. Mater. Chem. Front. 2020, 4, 1089-1104.

6. Wen, J. T.; Roper, J. M.; Tsutsui, H. Polydiacetylene supramolecules: Synthesis, characterization, and emerging applications. Ind. Eng. Chem. Res. 2018, 57, 9037-9053.

7. Pham, T. C.; Kim, H. S.; Lee, S. Polydiacetylenes functionalized with chelidamic acid and 2,2'-dipicolylamine for colorimetric responses to cadmium ions. Bull. Korean Chem. Soc. 2020, DOI: 10.1002/bkcs. 12177

8. Khanantong, C.; Charoenthai, N.; Wacharasindhu, S.; et al. Achieving reversible thermochromism of bisdiynamide polydiacetylene via self-assembling in selected solvents. Colloids and Surfaces A 2020, 603, 125225.

9. Pankaew, A.; Traiphol, N.; Traiphol, R. Tuning the sensitivity of polydiacetylene-based colorimetric sensors to UV light and cationic surfactant by co-assembling with various polymers. Colloids and Surfaces A 2021, 608, 125626.

10. Deng, J. L.; Guo, C. X.; Lu, W. S.; Liu, T.; Jiang, L.
Diacetylene vesicles-a biomolecular recognition device based on molecular assembly. Progress in Chemistry 2006, 18, 1397-1408.

11. Lee, S.; Kim, J.-Y.; Chen, X.; Yoon, J. Recent progress in stimuli-induced polydiacetylenes for sensing temperature, chemical and biological targets. Chem. Commun. 2016, 52, 9178-9196.

12. Wang, D.; Yan, J.; Wang, J. Polydiacetylene liposomes with phenylboronic acid tags: a fluorescence turn-on sensor for sialic acid detection and cell-surface glycan imaging. Nanoscale 2018, 10, 4570-4578.

13. Ahn, D. J.; Lee, S.; Kim, J.-M. Rational design of conjugated polymer supramolecules with tunable colorimetric responses. Adv. Funct. Mater. 2009, 19, 1483-1496.

14. Jiang, L. X.; Luo, J.; Dong, W. J.; et al. Development and evaluation of a polydiacetylene based biosensor for the detection of H5 influenza virus. Journal of Virological Methods 2015, 219, 38-45.

15. Jae-Pil, J.; Eunae, C.; Deokgyu, Y.; et al. Label-free colorimetric detection of influenza antigen based on an antibody-polydiacetylene conjugate and its coated polyvinylidene difluoride membrane. Polymers 2017, 9, 127.

16. Zhou, C.; You, T.; Jang, H.; Ryu, H.; et al. Aptamer-conjugated polydiacetylene colorimetric paper chip for the detection of bacillus thuringiensis spores. Sensors 2020, 20, 3124.

17. Shi, L. S.; Wu, Q. P.; Wu, H. Q.; et al. Application status and future development trend of food preservatives in my country. Food Research and Development 2008, 29, 157-161.

18. Wu, Z. X. Simultaneous determination of 5 preservatives and 2 sweeteners in cakes by high performance liquid chromatography. Agricultural Engineering 2013, 3, 80-82.

19. Mihyar, G. F.; Yousif, A. K.; Yamani, M. I. Determination of benzoic and sorbic acids in labaneh by high-performance liquid chromatography. J. Food Compos. Anal. 1999, 12, 53-61.

20. Yang, X. F.; Yang, K. Q.; Han, M.; et al. Determination of 7 preservatives in condiments by gel permeation chromatography purification-gas chromatography. Chinese Condiments 2011, 36, 107-110.

21. Pan, Z.; Wang, L.; Mo, W.; Wang, C.; Hu, W.; Zhang, J. Determination of benzoic acid in soft drinks by gas chromatography with on-line pyrolytic methylation technique. Anal. Chim. Acta 2005, 545, 218-223.

22. Xue, L.; Chen, L.; Dong, J.; et al. Dispersive liquid-liquid microextraction coupled with surface enhanced Raman scattering for the rapid detection of sodium benzoate. Talanta 2019, 208, 120360.

23. Li, X.; Liu, W.; Yue, X.; et al. A competitive immunoassay using hapten-modified polydiacetylene vesicles for homogeneous and 
sensitive detection of sodium benzoate. Sensors \& Actuators 2018, 258, 1060-1065. 